\title{
Droplet manipulation in a microfluidic chamber with acoustic radiation pressure and acoustic streaming
}

Yin Nee Cheung $*^{\mathrm{a}}$, Nam Trung Nguyen ${ }^{\mathrm{b}}$, Teck Neng Wong ${ }^{\mathrm{a}}$

a School of Mechanical and Aerospace Engineering, Nanyang Technological University, Singapore

*Email: mailccheung@gmail.com

b Queensland Micro- and Nanotechnology Centre, Griffith University, Brisbane QLD 4111, Australia

\begin{abstract}
The present paper reports a novel manipulation method for droplets using acoustic radiation pressure and acoustic streaming. In an acoustic field, droplets deform, oscillate and move in a wide range of applied frequencies. The behavior of a droplet depends on droplet size, acoustic field and interfacial tension between the two phases. The acoustic field is controlled by the voltage and frequency of the piezoelectric actuator. The results demonstrate a method for lowfrequency acoustic actuation of droplets in a microfluidic environment.
\end{abstract}




\section{Introduction}

The manipulation of droplets in microfluidic devices involves techniques to overcome forces that prevent the droplets to move and to deform such as friction forces and capillary forces. Acoustic energy has been demonstrated as a useful tool for the manipulation of droplets. Among the different configurations of acoustic actuation, the two common methods are acoustic levitation of droplets in air $^{1-13}$ and surface acoustic wave (SAW) for droplet on a planar surface or in a microfluidic device ${ }^{14-25}$. Evaporation is a major problem for the above two methods as droplets are manipulated in an open environment. ${ }^{5,18}$ Resonance cavity is another technique for liquid manipulation in microchannels using a tunable pressure source ${ }^{26}$. These devices can perform droplet positioning, merging, splitting, and sorting within an open microfluidic platform.

A substantial acoustic radiation force resulted from an intense ultrasonic field between an acoustic emitter and a reflector could levitate and deflect a droplet placed in this field. Periodic shape oscillations with different internal acoustic streaming patterns were reported for varying applied voltages and frequencies of the piezoelectric actuator. ${ }^{1,7-10}$ The non-uniform acoustic radiation force acts on the levitated droplet and causes it to deform. The interaction between the acoustic radiation pressure, surface tension and gravity leads to oscillation, translational vibration and rotation ${ }^{1,7-10}$ Various internal acoustic streaming patterns result from the interaction between the droplet fluid and the vibrating fluid-fluid interface under acoustic levitation. ${ }^{1,9}$ The levitation of droplets has found applications in contactless transport and handling in air. ${ }^{11-13}$ Adjusting the driving voltage of the piezoelectric transducer controls the levitation potential and allows for continuous planar transport. This method is useful for contact free handling of solids with different shapes and contact free handling (transportation, merging 
and atomization) of reactive or under-cooled liquids. Another application of droplet levitation is the analysis of surface rheological properties (equilibrium surface tension, Gibbs elasticity and surface dilatational viscosity) of a droplet. ${ }^{2,4}$

Surface acoustic wave (SAW) has been incorporated in microfluidic devices for directional transportation of droplets, cells and organisms, ${ }^{14,16,18,21-22,24-25}$ for handling a small liquid including mixing, sorting, jetting and atomization ${ }^{14,15,17-20,25}$ as well as for cell lysis ${ }^{23}$. The actuation is based on "leaky SAW" generated when the acoustic wave refracts at the interface between the solid substrate and the fluid. The longitudinal waves provide a force in their propagation direction and induce fluid flow within the liquid. The reflection of the wave at the boundary induces internal streaming in the confined liquid. The acoustic force breaks the droplet shape (left-right) symmetry and causes a contact angle hysteresis between the front and rear leading to the displacement of the droplet. SAW energy and direction can be controlled by designing interdigitated transducer with different configurations. ${ }^{18,22,24}$ Droplet transportation by SAW is triggered by droplet deformation that is caused by a combination of radiation pressure and acoustic streaming inside the droplet. ${ }^{16}$ In addition, the displaced droplet exhibits complicated creeping and jumping behavior along the movement direction. ${ }^{16,21}$

The present paper demonstrates a novel manipulation method for droplets in a microfluidic chamber utilizing acoustic radiation pressure and acoustic streaming. Acoustic actuation is applied from a piezoelectric transducer through a polydimethylsiloxane (PDMS) membrane. The droplet interface oscillates in different modes with various internal streaming patterns. Depending on the applied frequency, the droplet can move forward or backward and deform into 
different shapes. Droplet deformation leads to a curvature hysteresis between the front and rear of the droplet, which may trigger the motion of the droplet. This phenomenon resembles the contact angle hysteresis for a droplet moving on a plane substrate. ${ }^{27-34}$ The observed displacement of the droplet is proportional to the applied voltage. The use of surfactant in the continuous phase increases the effectiveness of acoustic actuation. However, there is an upper limit for surfactant concentration, beyond which the droplet displacement decreases. Droplet trajectories are analyzed for obtaining the velocity from the displacement data. The deformation of an oscillating droplet reveals the shape evolution during actuation process.

\section{Device design and experimental setup}

The microfluidic device used in our experiment was fabricated with soft-lithography technique. ${ }^{35}$ Figure 1 shows the design of the microfluidic device. In order to generate droplets with controlled volumes and for positioning the droplets into the acoustic chamber, T-junctions with bypass channels are adopted. ${ }^{36}$ A $135-\mu$ m thick layer of SU8 photoresist (SU8-100, MicroChem Corp.) was spin coated onto a silicon wafer. UV-lithography transferred the patterns to the photoresist. The developed photoresist with the microchannel patterns was used as a master mold for replicating PDMS devices. A piezoelectric disk with a diameter $31.8 \mathrm{~mm}$ (T216-A4NO-373X, Piezo Systems, Inc.) was embedded in the PDMS device using adhesive spacers. The gap between the bottom of the piezoelectric disk and the top of the microchannel is $1.1 \mathrm{~mm}$. A mixture of PDMS pre-polymer and its hardener (Sylgard 184, Dow Corning Corp.) was prepared in a proportion of 10:1 by weight. The PDMS mixture was then cured in an oven at $80{ }^{\circ} \mathrm{C}$ for 2.5 hours. The cured PDMS was peeled off from the silicon wafer and then cut to size and punched 
with inlet and outlet holes. The PDMS device was then bonded to glass slide coated with a thin layer of PDMS with the assistance of oxygen plasma. A sinusoidal electric signal conditioned by a signal generator (AFG320, Tektronix, Inc.) and a power amplifier (790 Series, PCB Piezotronics, Inc.) drove the piezoelectric disk.

The dispersed and continuous phases in the experiment are deionized (DI) water and mineral oil (M5904, Sigma Aldrich Co.) mixed with $0.5 \%$ w/w (by weight), 1\% w/w, 2\% w/w and 3\% w/w of Span 80 (S6760, Sigma Aldrich Co.), respectively. Interfacial tensions between mineral oil and DI water were measured using a tensiometer (FTA200, First Ten Ångstroms, Inc.). The measured values of the interfacial tensions are $4.67 \mathrm{mN} / \mathrm{m}(0.5 \% \mathrm{w} / \mathrm{w}), 4.34 \mathrm{mN} / \mathrm{m}(1 \% \mathrm{w} / \mathrm{w})$, $4.23 \mathrm{mN} / \mathrm{m}(2 \% \mathrm{w} / \mathrm{w}), 4.24 \mathrm{mN} / \mathrm{m}(3 \% \mathrm{w} / \mathrm{w})$. The viscosity of the mineral oils with surfactant Span 80 was measured with a rheometer (DHR-2, TA Instruments, Inc.). As the standard deviation of the measured viscosity for the different surfactant concentrations mentioned above is less than $5 \%$, the average viscosity value of $26.94 \mathrm{mPa} . \mathrm{s}$ is used. Before each experiment, the aqueous droplet generated from the $\mathrm{T}$-junction was positioned to the middle of the rectangular chamber as shown in Fig. 1. A high speed camera (FASTCAM-APX RS, Photron, Inc.) attached to an inverted microscope (Eclipse Ti, Nikon Ltd.) was used to capture the motion of the droplet. The dynamics and kinematics of the droplet motion were evaluated from recorded images using the public-domain processing software ImageJ. ${ }^{37}$ To trace the flow patterns, both liquid phases were mixed with dry fluorescent particles (nominal diameter $7 \mu \mathrm{m}, 35-2 \mathrm{~B}$, Thermo Fisher Scientific, Inc.).

\section{Results and discussion}


Depending on the applied actuation frequency, the droplet exhibits different shapes. The initial circular shape (Fig. 2(a)) gradually changes into an oblate shape (Fig. 2(b)) when actuated at a sinusoidal frequency of $50 \mathrm{~Hz}$. The displacement and geometric parameters of the droplet were evaluated from the start of the acoustic actuation $(t=0)$. The data include the deformations in the $x$ and $y$ direction $(\Delta x, \Delta y)$ and the location of the center point of the droplet $\left(x_{c}, y_{c}\right)$ with $x_{c}=$ $\left(x_{\min }+x_{\max }\right) / 2$ and $y_{\mathrm{c}}=\left(y_{\min }+y_{\max }\right) / 2$.

\subsection{Frequency dependent droplet translocation and flow patterns under acoustic actuation}

In our device configuration, deformation, shape oscillation and translocation direction depend on the applied acoustic frequency. An operation range from $50 \mathrm{~Hz}-6 \mathrm{kHz}$ was applied to investigate the effect of frequency. Images were captured at a rate of 5 frames per second (fps) with a shutter speed of $1 / 3000 \mathrm{~s}$ and processed with the ImageJ software ${ }^{37}$ for obtaining the droplet outline. Fig. 3 shows the displacement trajectories of a $920 \mu \mathrm{m}$ droplet driven by eight different frequencies and a voltage of $\pm 144 \mathrm{~V}$. The arrows indicate the translocation direction. The droplet outlines are plotted for every 2 seconds over duration of 40 seconds. At $50 \mathrm{~Hz}$, the droplet deforms into an oblate shape with elongation in the $x$ direction and moves forward, away from the acoustic source. However, as the frequency increases, the shape gradually elongates in the $y$ direction, and finally moves backward towards the acoustic source at $2 \mathrm{kHz}$. The transition of the deformation and the motion direction is at around $1.7 \mathrm{kHz}$, where the droplet oscillates and shows little movement. 
Dry particles with a nominal diameter of $7 \mu \mathrm{m}$ (35-2B, Thermo Fisher Scientific Inc.) were mixed into DI water at a concentration of $0.7 \%$ w/w. Mineral oil (M5904) with 1\% w/w Span 80 was used as the continuous phase. Flow patterns shown in Fig. 4 are generated by superimposing 6 to 15 images. The supplementary videos (S1-S3) show the internal flow fields of the same droplet under frequencies of $50 \mathrm{~Hz}, 1.6 \mathrm{kHz}$ and $4.5 \mathrm{kHz}$. Fig. 4(a,b,c) shows the acoustic streaming field with four vortices inside a droplet actuated at $50 \mathrm{~Hz}, 500 \mathrm{~Hz}$ and $1 \mathrm{kHz}$, respectively. Acoustic streaming is a nonlinear phenomenon which is resulted from the propagation of acoustic wave in the fluid. ${ }^{38}$ The two vortices on the lower part of the droplet provide the main driving force to push the droplet forward as they rotate with a higher speed and occupy a larger proportion (S1). As the frequency increases to $1.5 \mathrm{kHz}, 1.6 \mathrm{kHz}$ and $2 \mathrm{kHz}$, the upper two vortices occupy a larger proportion and become the main driving force (Fig. 4 (d,e,f), S2). Hence, the droplet starts to move backward. If the frequency increases up to $4.5 \mathrm{kHz}, 5 \mathrm{kHz}$ and $6 \mathrm{kHz}$, there are no obvious vortices inside the droplet (Fig. 4 (g,h,i), S3). Particles move from the top to the bottom and then to the top of the droplet through a circulation across the depth of the droplet. The droplet is driven towards the source.

Supplementary movies S4 and S5 show the flow field in both the droplet and the surrounding oil at $50 \mathrm{~Hz}$ and $2 \mathrm{kHz}$. For the $50 \mathrm{~Hz}$ case, vigorous streaming in the continuous phase around the bottom of the droplet and an expanding flow around the top of the droplet are observed. In addition, particles move in a rolling motion around the droplet boundary. At $2 \mathrm{kHz}$, no streaming was observed in the continuous phase. The particles move gently along the $y$ direction. Droplet actuation in this case could be regarded as internally driven. 


\subsection{Droplet kinematics}

\subsubsection{Displacement}

Droplet displacement was obtained by extracting the $y$ position of the center point of the droplet $\left(x_{c}, y_{c}\right)$ from a sequence of around 200 images. Figure 5 shows the displacement versus time of droplets actuated at different applied frequencies, voltages, diameters and surfactant concentrations.

Droplet displacements in the $y$ direction for the frequency range of $50 \mathrm{~Hz}-2 \mathrm{kHz}$ are shown in Fig. 5(a). The displacement curves show the response of the droplet motion with respect to the applied acoustic frequency showing the feasibility of droplet motion control by frequency.

A forward motion away from the acoustic source is observed when the droplet is actuated at 50 $\mathrm{Hz}$ (S6). According to Fig. 5, a droplet moves faster in the initial stage and gradually moves with a lower velocity. The slope of the displacement and consequently the velocity of the droplet increases with increasing voltage, Fig. 5 (b).

The balance between the acoustic energy and the energy loss through acoustic streaming and droplet deformation determines the behavior of droplet kinematics. An increase in the induced energy leads to a larger asymmetry of the droplet (in terms of curvature hysteresis of the front and the rear parts of the droplet) which would contribute to a faster droplet motion and thus a larger displacement. Fig. 5 (c) shows that a larger droplet has a larger overall displacement ( $2200 \mu \mathrm{m}$ for $d=960 \mu \mathrm{m})$ as compared to a smaller one $(\sim 1600 \mu \mathrm{m}$ for $d=660 \mu \mathrm{m})$. Under the 
same actuation conditions, a smaller droplet deforms less than a larger droplet. Surface tension hinders droplet deformation and absorbs part of the induced acoustic energy leading to a slower motion and a shorter displacement.

Fig. 5 (d) shows the effect of surfactant concentration on the droplet motion. The increase of Span 80 concentration from $0.5 \% \mathrm{w} / \mathrm{w}$ to $1 \% \mathrm{w} / \mathrm{w}$ facilitates droplet motion for a longer distance. Besides reducing the interfacial tension, the increase of surfactant concentration causes a reduction in the frictional force between the droplet and the channel wall due to change in wetting property of the oil film separating them. However, further increasing Span 80 concentration beyond $1 \% \mathrm{w} / \mathrm{w}$ causes a reduction in the total displacement.

\subsubsection{Energy consideration}

The total energy for driving a droplet over a period of time $t$ is estimated as:

$$
E_{t}=E_{a c}-E_{f}-E_{d}
$$

where $E_{a c}$ is the induced acoustic energy, $E_{f}$ is the energy for overcoming friction and $E_{d}$ is the energy for deforming the droplet. In the current investigation, we take the total displacement in the $y$ direction $D_{y, t}$ over a given period of time $t$ as the dependent variable and which can be related to $E_{t}$ as:

$$
E_{t}=\left\langle F_{a c}\right\rangle * D_{y, t} \sim D_{y, t}
$$

where $\left\langle F_{a c}\right\rangle$ is the time-averaged driving force which is assumed to be constant over the period $t$. Combining (1) and (2) results in:

$$
D_{y, t} \sim E_{a c}-E_{f}-E_{d} .
$$


A time period of $t=35 \mathrm{~s}$ is taken for the investigation. The total distance travelled over this time period, $D_{y, 35}$, is investigated as a function of various actuation parameters such as voltage $V$, frequency $f$, droplet diameter $d$, and surfactant concentration $S$.

\section{Acoustic energy}

The induced acoustic energy could be determined from the time-averaged acoustic energy density $e_{a c}=4 E_{a c} / \pi d^{2} h$, assuming the droplet has the shape of a disc with a diameter $d$ and a height $h$. The acoustic wave acts as a harmonic oscillator. Fluid kinetic energy and the pressurerelated potential energy converted into each other periodically during the oscillation:

$$
e_{a c} \propto \frac{p_{a}^{2}}{K_{0}},
$$

where $p_{a}$ is the amplitude of the pressure wave and $K_{0}$ is the bulk modulus of the liquid. ${ }^{39-40}$ For piezoelectric actuation, a linear relationship exists between the applied peak-to-peak voltage $U_{p p}$ and the induced acoustic pressure amplitude $p_{a}:^{41}$

$$
p_{a} \propto U_{p p}
$$

hence,

$$
e_{a c} \sim U_{p p}^{2}
$$

However, Figure 6 (a) shows a linear relationships between the displacement $D_{y, 35}$ and the applied voltages of droplets with different sizes. The displacement-voltage relationship follows an increasing trend with a similar slope. The deviation from the second order relationship estimated in (6) may be caused by energy losses as discussed in the following section. 


\section{Frictional energy loss}

Part of the induced acoustic energy is dissipated as the acoustic streaming flow in both the continuous and the dispersed phases. For an oscillatory flow with amplitude $U_{0}$ and frequency $\omega$, the Stokes layer varies with the length scale $L$ along the boundary. The rectification between the nonlinear inertial term and the oscillatory flow results in a steady inertial force. This steady inertial force is balanced by steady viscous forces resulting in a steady boundary-driven streaming with a velocity: ${ }^{42}$

$u_{s, I} \sim \frac{U_{0}^{2}}{\omega L}$

The acoustic boundary-driven slip velocity outside the Stokes layer is: ${ }^{42}$

$u_{s, I I} \sim-3 U_{0}\left(\frac{d U_{0}}{d x} / 4 \omega\right)$

The streaming flow velocity increases with increasing applied acoustic voltage $V^{43-44}$ Hence, both the frequency and the applied voltage determine the energy dissipation in the form of kinetic energy of the flow. Fig. 6 (b) shows a linearly decreasing relationship between the displacement and the applied frequency. As mentioned in Section 3.1, the droplet vibrates in various modes flow patterns over a range of frequency. The directional motion of the droplet is governed by the streaming pattern inside the droplet. At the transition between different streaming modes, the droplet slows down and changes its direction, Fig. 3.

\section{Energy of droplet deformation}

Let $e_{d}$ be the energy per unit volume required for deforming the droplet with an induced acoustic energy $4 E_{d} / \pi d^{2} h$ with: 
$e_{d} \sim \Delta p$,

where $\Delta p$ is the average difference in Laplace pressure between the advancing and the receding ends of the droplet over a time period:

$\Delta p \sim \gamma\left\langle\frac{1}{\Delta R}\right\rangle$

where $\gamma$ is the interfacial tension, and the curvature difference is determined as:

$\frac{1}{\Delta R}=\frac{1}{T} \int_{0}^{T}\left(\frac{1}{R_{a d v}}-\frac{1}{R_{r e c}}\right) d t$

where $T$ is the period of oscillation, $R_{a d v}$ and $R_{r e c}$ are the radii of curvature for the advancing and the receding ends of the droplet. ${ }^{30-31}$ Combining (9) and (10) leads to:

$e_{d} \sim \gamma\left\langle\frac{1}{\Delta R}\right\rangle$

Fig. 7 (a) shows a decreasing trend between $D_{y, 35}$ and the surfactant concentration. The surfactant concentrations used in our experiments are near the critical micelles concentration. The interfacial tension variation is around $10 \%$ for the range of concentrations used. A reduction in the surfactant concentration promotes droplet motion according to (12) as less energy is required for droplet deformation. In addition, the surfactant reduces the friction in the oil film between the droplet and the channel wall. As the concentration increases up to 2 and $3 \% \mathrm{w} / \mathrm{w}$, the droplet motion becomes less stable and displacement curves exhibit ripples. The droplet continuously slips backward during the course of its forward motion. Difference between the advancing and the receding curvatures are extracted by best fitting a portion of the front and the rare parts of the 
droplets to a circle. The droplet with diameter $660 \mu \mathrm{m}$ was investigated and the variations at different voltages are shown in Fig. 7(b). The difference in curvature is oscillating at a rate equal to the applied frequency. The time-averaged value of the difference in curvatures is positive indicating that the net motion is in forward direction. In addition, an increase in the applied voltage causes an increase in the peak-to-peak value of the curvature difference.

\section{Total energy}

The total energy required for droplet displacement is:

$$
E_{t}=\frac{1}{4}\left(e_{a c}-e_{f}-e_{d}\right) \pi d^{2} h
$$

Fig. 8 (a) shows an increasing trend between $D_{y, 35}$ and the droplet size $d$. Larger droplets collect more acoustic energy and thus exhibit a larger displacement. However, droplet deformation is more obvious for larger droplets as discussed later in Section 3.2.4. Thus, energy dissipation is higher and causes an upper bound for droplet displacement, Fig. 8 (b).

\subsubsection{Droplet velocity}

Droplet velocity is calculated based on the sampling theorem and the use of a noise-filtered differentiator to perform true differentiation of the displacement data. ${ }^{45}$ The experimental data as obtained, $f(t)$, can be recovered using the following interpolator:

$$
\hat{f}(t)=\sum_{K=-\infty}^{\infty} f(K \hat{T}) \hat{h}(t-K \hat{T})
$$


where $\hat{f}$ is the recovered experimental data, $K$ is an arbitrary number, $\hat{T}$ is the sampling interval and $\hat{h}$ is the truncated impulse response of the filter. The velocity $v(t)$ of the droplet could be obtained by calculating the first derivative of (14) as:

$v(t)=\frac{d \hat{f}}{d t}=\sum_{K=-\infty}^{\infty} f(K \hat{T}) \frac{d \hat{h}(t-K \hat{T})}{d t}$

Figure 9 shows the original and the recovered displacement curves for a droplet with a diameter of $960 \mu \mathrm{m}$ in a medium of mineral oil with $0.5 \% \mathrm{w} / \mathrm{w}$ Span 80 . The recovered curves are almost identical to their original curves. The velocities of the droplets are then calculated based on this theory. Figures 10 (a) and (b) show the velocity of droplets with diameters of $960 \mu \mathrm{m}$ and 660 $\mu \mathrm{m}$, respectively. When acoustic actuation starts to impart on the droplet, the droplet accelerates to a maximum velocity and then decelerates to lower level, Fig. 10. Droplet velocities are proportional to the acoustical input energy. Maximum velocity $v_{y, \max }$ increases with increasing applied voltage, Fig. 8(b). Similar results have been reported by Baudoin et al. ${ }^{21}$ For larger droplets, increase in voltage causes increasing deformation and consequently a reduction in the energy for droplet transportation and thus a lower velocity.

\subsubsection{Drop shape deformation and oscillation}

Since droplet oscillation promotes its mobility, it is worth to study droplet oscillation under acoustic actuation. Values of the total droplet deformation in the $x$ and $y$ directions $(\Delta x, \Delta y)$ and droplet oscillation in the $y$ direction, change of $y_{c}$ over time, were extracted from a sequence of images. Results for a droplet with diameter of $960 \mu \mathrm{m}$ are plotted in Figs. 11 and 12. Figure 11 
shows that the oscillating droplet shapes change under different applied voltages. In general, the ratio of droplet shape change $(\Delta y / \Delta x)$ is the same for all applied voltages. A higher applied voltage causes a larger deformation. The rate of shape change in the initial stage is higher to impart a momentum to the droplet leading to its acceleration. This rate slows down and reaches a more stable value in the later stage. The shape change recoils back and maintains a constant value after reaching the maximum deformation (indicated by the arrows in Fig. 11). Fig. 12 shows that the droplet oscillates at $50 \mathrm{~Hz}$ which is the driving frequency. The amplitude of oscillation is directly proportional to the applied voltage. During the oscillation, the droplet continuously prolongs in the $x$ direction $(\Delta x)$ and contracts in the $y$ direction $(\Delta y)$ until the oblate shape is reached.

\section{Conclusions}

A novel method of droplet manipulation in a microfluidic chamber with acoustic radiation pressure and acoustic streaming is presented in this paper. Droplets exhibit deformation and motion in a range of driving frequencies $(50 \mathrm{~Hz}-6 \mathrm{kHz})$. Particles in both the dispersed and the continuous phases are used for visualizing acoustic streaming. The flow patterns indicate that droplet motion is facilitated by the acoustic streaming inside the droplet. The flow pattern is related to the direction of the droplet motion. Droplet displacement was analyzed and velocities were obtained based on the sampling theorem and a noise-filtered differentiator. The results indicate that droplet displacements are in general proportional to the applied voltage. Droplet oscillation and deformation facilitate its motion. In response to the acoustic actuation, a droplet deforms and accelerates to a maximum velocity and then decelerates to a lower level. The effect of surfactant concentration and the corresponding change in interfacial tension was also studied. 
If the surfactant concentration is relatively low $(0.5-1 \% \mathrm{w} / \mathrm{w})$, an increase in surfactant concentration improves the motion of the droplet. However, a higher concentration $(2-3 \% \mathrm{w} / \mathrm{w})$ hinders the motion of the droplet. The work reported here demonstrates a new method for a low frequency acoustic actuation of droplets in a microfluidic environment.

\section{Acknowledgements}

The authors would like to thank Prof. Haiqing Gong for the use of his microscope and high speed camera. The authors gratefully acknowledge research support from the Singapore Ministry of Education Academic Research Fund Tier 2 research grant MOE2011-T2-1-036.

\section{References}

1. E. Trinh and T. G. Wang, J. Fluid Mech., 1982, 122, 315-338.

2. E. H. Trinh, P. L. Marston and J. L. Robey, J. Colloid Interface Sci., 1988, 124, 95-103.

3. E. H. Trinh and J. L. Robey, Phys. Fluids, 1994, 6, 3567-3579.

4. Y. Tian, R. G. Holt and R. E. Apfel, J. Colloid Interface Sci., 1997, 187, 1-10.

5. A. L. Yarin, G. Brenn, O. Kastner, D. Rensink and C. Tropea, J. Fluid Mech., 1999, 399, 151-204.

6. K. Ohsaka and E. H. Trinh, Phys. Rev. Lett., 2000, 84, 1700-1703.

7. W. J. Xie and B. Wei, Phys. Rev. E: Stat. Phys., Plasmas, Fluids, Relat. Interdiscip. Top., 2004, 70, 046611.

8. C. L. Shen, W. J. Xie and B. Wei, Phys. Rev. E: Stat. Phys., Plasmas, Fluids, Relat. Interdiscip. Top., 2010, 81, 046305.

9. C. L. Shen, W. J. Xie, Z. L. Yan and B. Wei, Phys. Lett. A, 2010, 374, 4045-4048. 
10. C. L. Shen, W. J. Xie and B. Wei, Phys. Lett. A, 2010, 374, 2301-2304.

11. N. Bjelobrk, M. Nabavi and D. Poulikakos, J. Appl. Phys., 2012, 112, 053510.

12. N. Bjelobrk, D. Foresti, M. Dorrestijn, M. Nabavi and D. Poulikakos, Appl. Phys. Lett., 2010, 97, 161904.

13. D. Foresti, M. Nabavi, M. Klingauf, A. Ferrari and D. Poulikakos, Proc. Natl. Acad. Sci. U. S. A., 2013, 110, 12549-12554.

14. A. Wixforth, C. Strobl, Ch. Gauer, A. Toegl, J. Scriba and Z. v. Guttenberg, Anal. Bioanal. Chem., 2004, 379, 982-991.

15. T. Frommelt, M. Kostur, M. Wenzel-Schäfer, P. Talkner, P. Hänggi and A. Wixforth, Phys. Rev. Lett., 2008, 100, 034502.

16. P. Brunet, M. Baudoin, O. Bou Matar and F. Zoueshtiagh, Phys. Rev. E: Stat. Phys., Plasmas, Fluids, Relat. Interdiscip. Top., 2010, 81, 036315.

17. M. K. Tan, J. R. Friend, O. K. Matar and L. Y. Yeo, Phys. Fluids, 2010, 22, 112112.

18. S. S. Wang, Z. J. Jiao, X. Y. Huang, C. Yang, N. T. Nguyen, Microfluid. Nanofluid., $2009,6,847-852$

19. T.-D. Luong, V.-N. Phan and N.-T. Nguyen, Microfluid. Nanofluid., 2011, 10, 619-625.

20. R. J. Shilton, L. Y. Yeo and J. R. Friend, Sens. Actuators, B, 2011, 160, 1565-1572.

21. M. Baudoin, P. Brunet, O. Bou Matar and E. Herth, Appl. Phys. Lett., 2012, 100, 154102.

22. Y. Ai and B. L. Marrone, Microfluid. Nanofluid., 2012, 13, 715-722.

23. J. Reboud, Y. Bourquin, R. Wilson, G. S. Pall, M. Jiwaji, A. R. Pitt, A. Graham, A. P. Waters and J. M. Cooper, Proc. Natl. Acad. Sci. U. S. A., 2012, 109, 15162-15167.

24. X. Ding, S.-C. S. Lin, B. Kiraly, H. Yue, S. Li, I.-K. Chiang, J. Shi, S. J. Benkovic and T. J. Huang, Proc. Natl. Acad. Sci. U. S. A., 2012, 109, 11105-11109. 
25. X. Ding, P. Li, S.-C. S. Lin, Z. S. Stratton, N. Nama, F. Guo, D. Slotcavage, X. Mao, J. Shi, F. Costanzo and T. J. Huang, Lab Chip, 2013, 13, 3626-3649.

26. S. M. Langelier, D. S. Chang, R. I. Zeitoun and M. A. Burns, Proc. Natl. Acad. Sci. U. S. A., 2009, 106, 12617-12622.

27. N.-T. Nguyen, Langmuir, 2013, 29, 13982-13989.

28. N.-T. Nguyen, G. Zhu, Y.-C. Chua, V.-N. Phan and S.-H. Tan, Langmuir, 2010, 26, 12553-12559.

29. L. Gao and T. J. McCarthy, Langmuir, 2006, 22, 6234-6237.

30. P. Brunet, J. Eggers and R. D. Deegan, Phys. Rev. Lett., 2007, 99, 144501.

31. L. Dong, A. Chaudhury and M. K. Chaudhury, Eur. Phys. J. E, 2006, 21, 231-242.

32. X. Noblin, A. Buguin and F. Brochard-Wyart, Eur. Phys. J. E, 2004, 14, 395-404.

33. P. Brunet, J. Eggers and R. D. Deegan, Eur. Phys. J.: Spec. Top., 2009, 166, 11-14.

34. D. Bonn, J. Eggers, J. Indekeu, J. Meunier and E. Rolley, Rev. Mod. Phys., 2009, 81, 739-805.

35. J. C. McDonald, D. C. Duffy, J. R. Anderson, D. T. Chiu, H. Wu, O. J. A. Schueller and G. M. Whitesides, Electrophoresis, 2000, 21, 27-40.

36. V. van Steijn, P. M. Korczyk, L. Derzsi, A. R. Abate, D. A. Weitz and P. Garstecki, Biomicrofluidics, 2013, 7, 024108.

37. W. S. Rasband, ImageJ, U.S. National Institutes of Health, Bethesda, Maryland, USA, http://imagej.nih.gov/ijl, 1997-2014.

38. A. H. Meng, N. T. Nguyen, R. M. White, Biomed. Microdev., 2000, 2, 169-174.

39. H. Bruus, Lab Chip, 2012, 12, 1578-1586.

40. R. Barnkob, P. Augustsson, T. Laurell and H. Bruus, Lab Chip, 2010, 10, 563-570. 
41. J. Dual and D. Möller, Lab Chip, 2012, 12, 506-514.

42. T. M. Squires and S. R. Quake, Rev. Mod. Phys., 2005, 77, 977-1026.

43. B.-G. Loh, S. Hyun, P. I. Ro and C. Kleinstreuer, J. Acoust. Soc. Am., 2002, 111, 875-883.

44. X. Y. Du, M. E. Swanwick, Y. Q. Fu, J. K. Luo, A. J. Flewitt, D. S. Lee, S. Maeng and W.

I. Milne, J. Micromech. Microeng., 2009, 19, 035016.

45. X.-S. Li, J. Yang and H. Liu, J. Eng. Mech. Div., Am. Soc. Civ. Eng., 1998, 124, 705-712. 


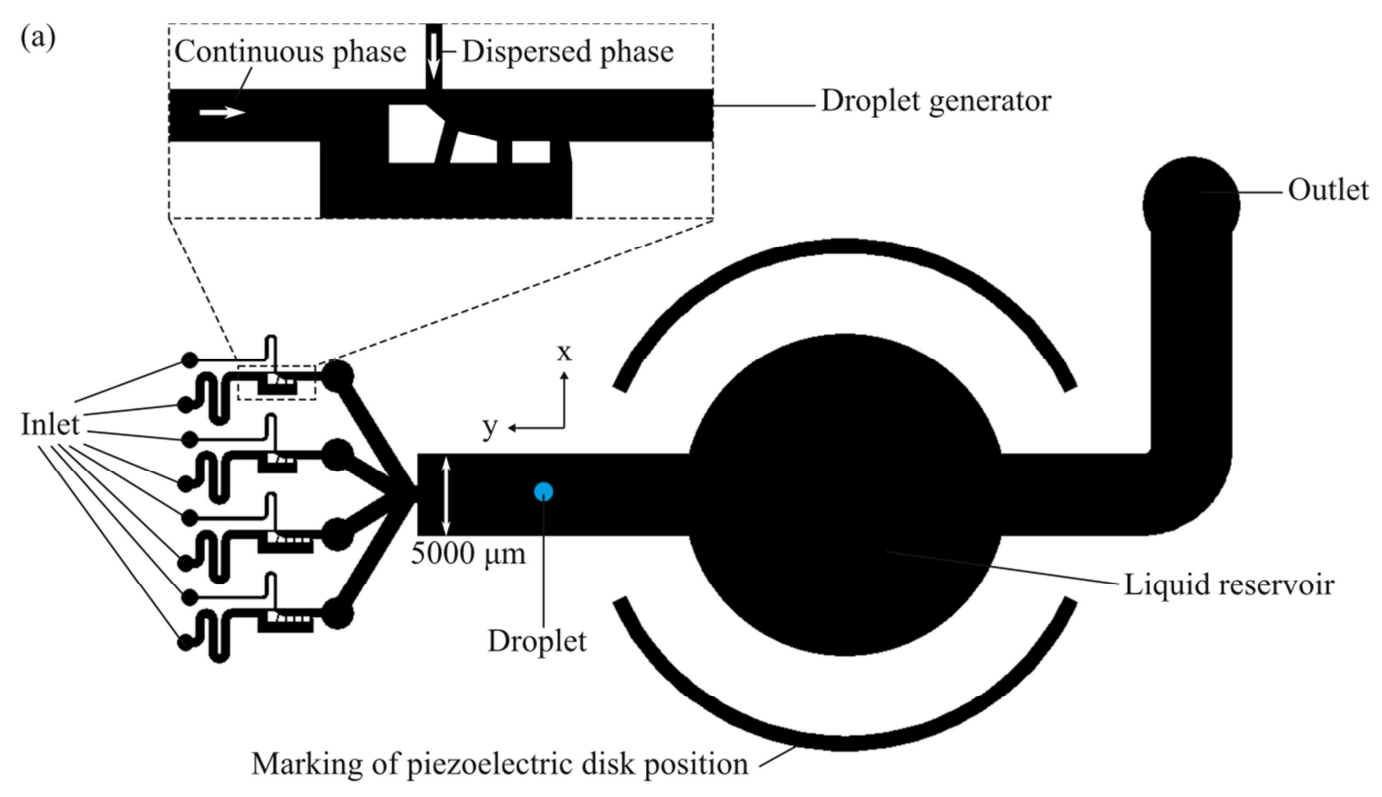

(b)

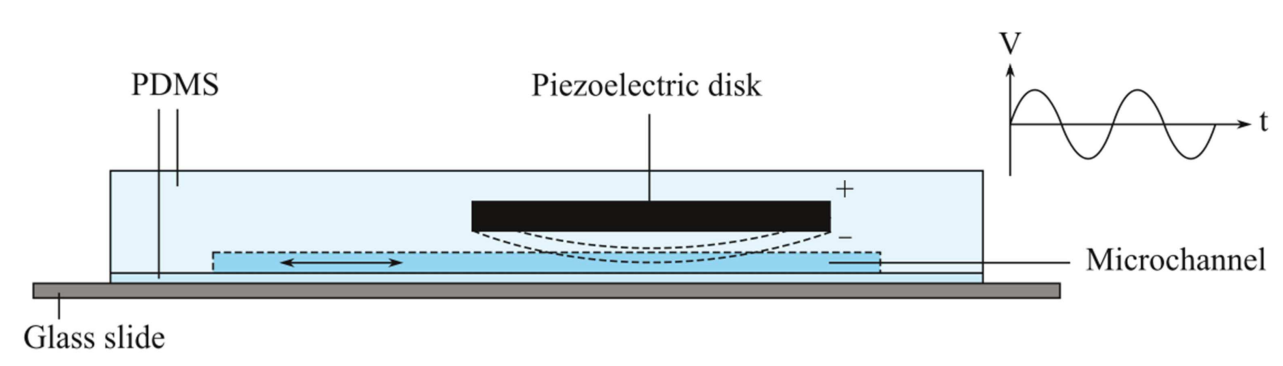

Fig. 1 (a) Device design for generating droplets with controlled volumes using T-junctions with bypasses (inset). The droplet generated is transported downstream to the rectangular chamber for experimentation. A piezoelectric disk of diameter $31.8 \mathrm{~mm}$ is embedded in the PDMS device. The piezoelectric disk has a free deflection of $\pm 119 \mu \mathrm{m}$ at a rated voltage of $\pm 180 \mathrm{~V}$. (b) Side view of the microfluidic device. Fluid inside the microchannel is actuated to oscillate in the transversal direction as indicated by the double arrow. 
(a)

\section{(b)}

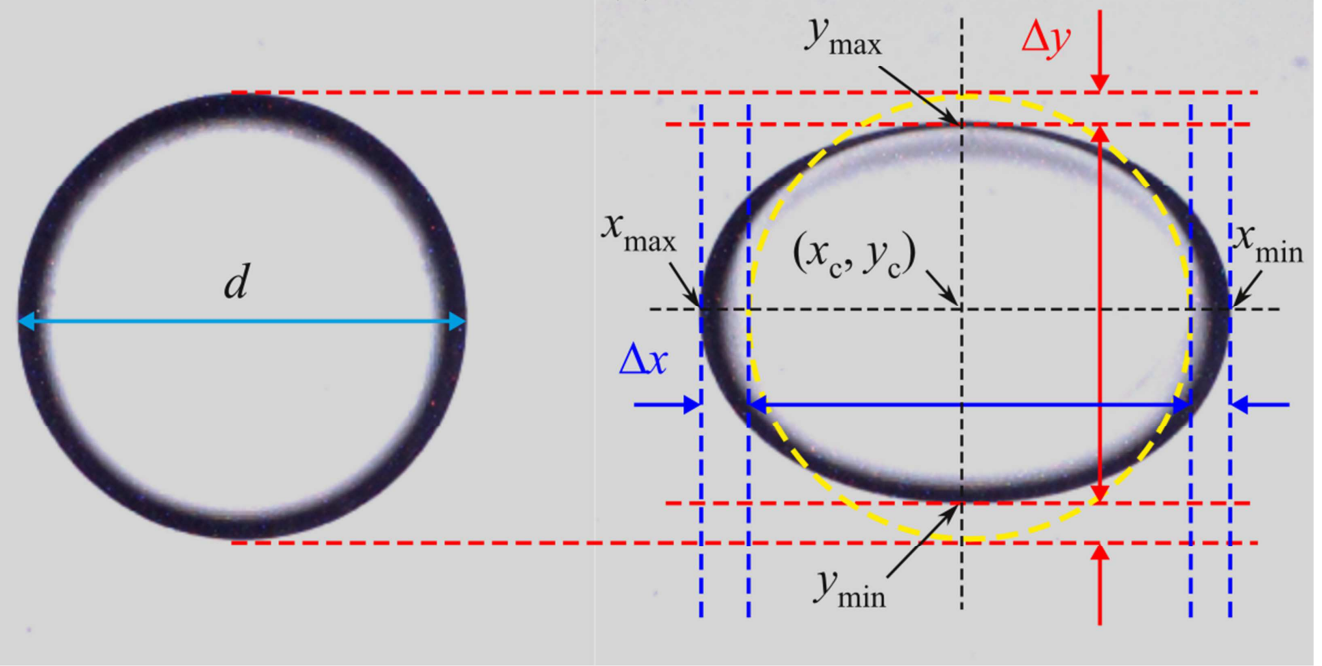

Fig. 2 (a) Droplet with a diameter $d$ before acoustic actuation. (b) Deformed droplet under acoustic actuation with a sinusoidal frequency of $50 \mathrm{~Hz}$ at $\pm 156 \mathrm{~V}$. The total deformation in the $x$ and $y$ directions are $\Delta x$ and $\Delta y$, respectively. The location of the center point of the droplet is $\left(x_{\mathrm{c}}\right.$, $\left.y_{\mathrm{c}}\right)$ 
(a)

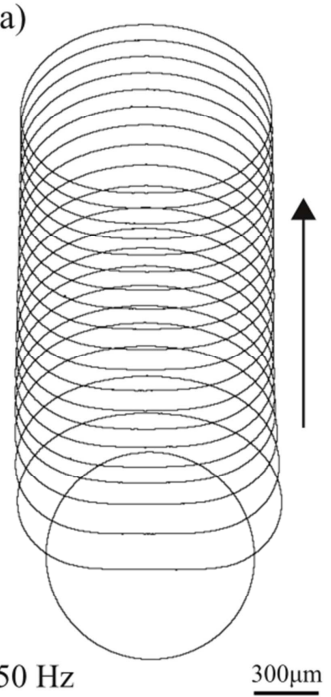

(e)

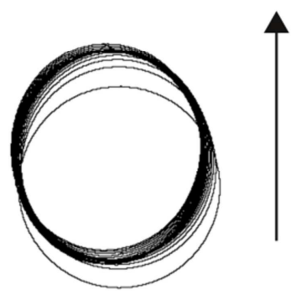

y

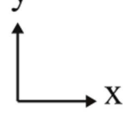

$1.6 \mathrm{kHz}$ (b)

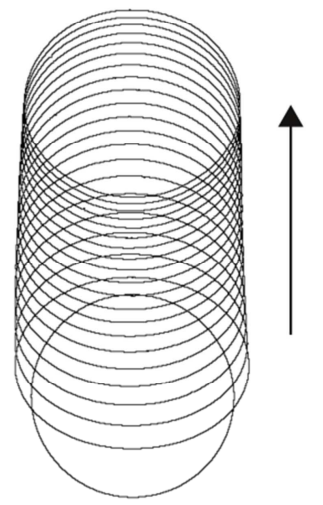

$300 \mu \mathrm{m} \quad 800 \mathrm{~Hz}$

(g)

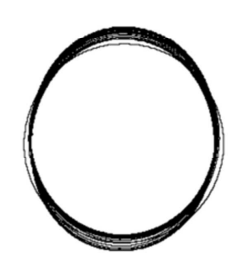

(f)

$300 \mu \mathrm{m} \quad 1.8 \mathrm{kHz}$

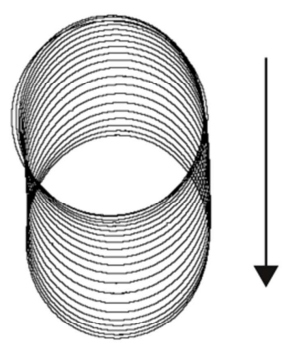

(d)
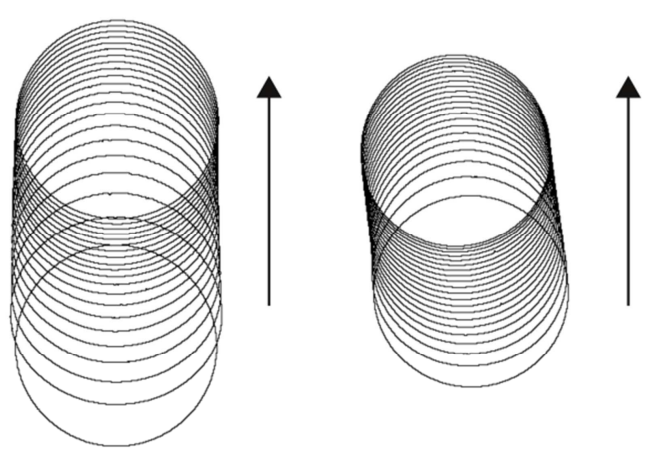

$300 \mu \mathrm{m} \quad 1.2 \mathrm{kHz}$

$\underline{300 \mu \mathrm{m}}$

(h)

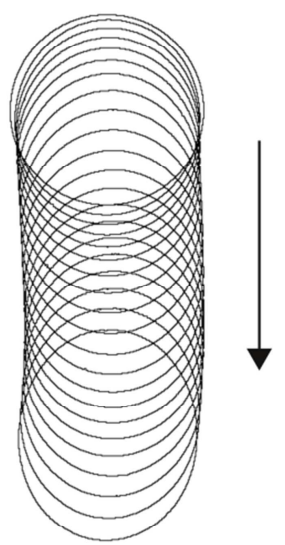

$\underline{300 \mu \mathrm{m}} \quad 2 \mathrm{kHz}$

$\underline{300 \mu \mathrm{m}}$

Fig. 3 Droplet displacement trajectories. Arrows indicate the displacement direction. The corresponding acoustic actuation frequency is indicated in each figure and the actuation voltage for all the trajectories is $\pm 144 \mathrm{~V}$. The droplet has a diameter $920 \mu \mathrm{m}$ and moves in mineral oil (M5904) with 0.5\% w/w Span 80. 
(a)
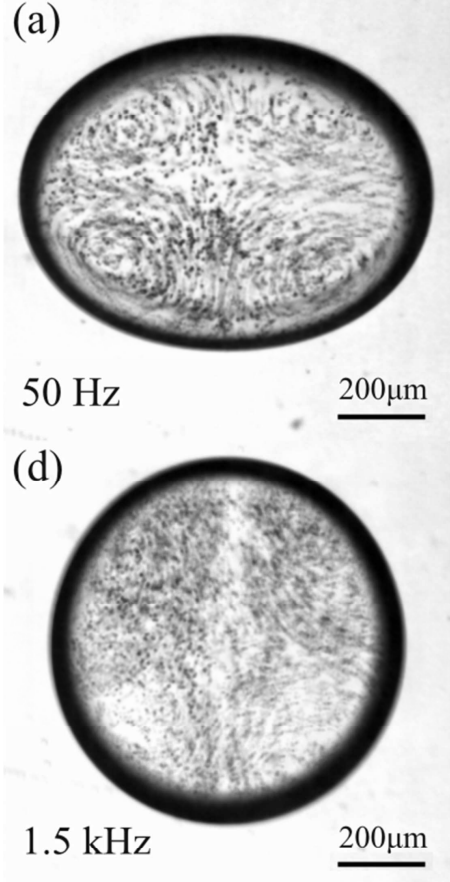

(g)

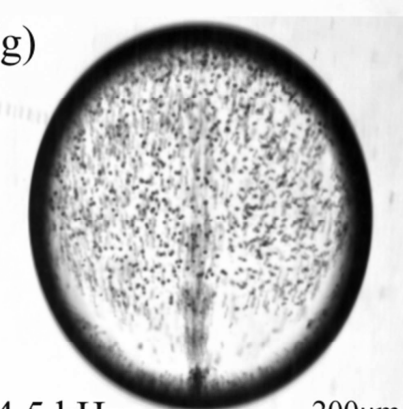

$4.5 \mathrm{kHz}$ (b)

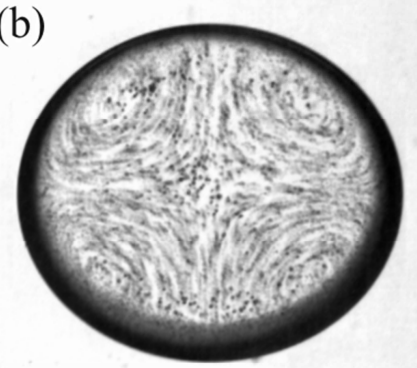

$500 \mathrm{~Hz}$

(e)

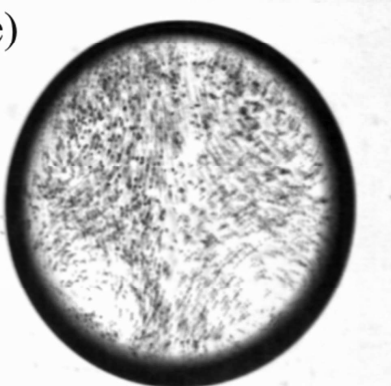

$1.6 \mathrm{kHz}$

(h)

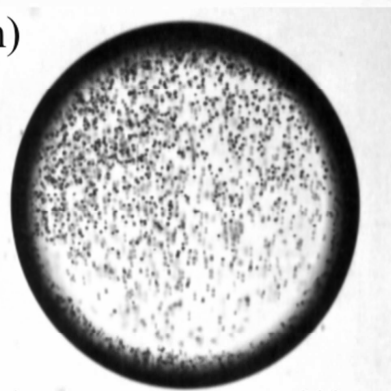

(c)

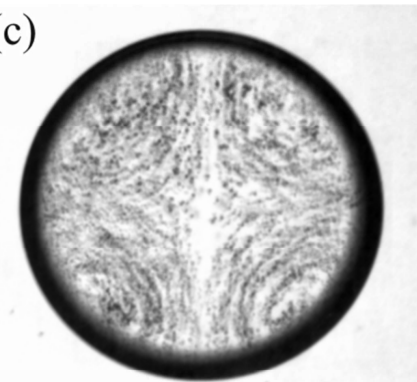

$1 \mathrm{kHz} \quad 200 \mu \mathrm{m}$

(f)

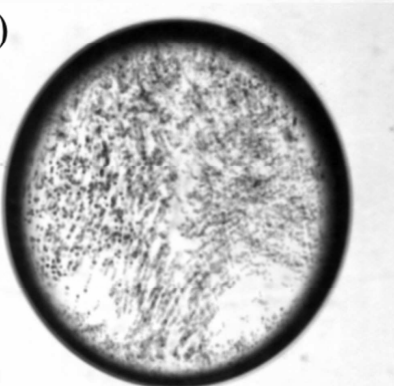

$2 \mathrm{kHz} \quad \underline{200 \mu \mathrm{m}}$

(i)

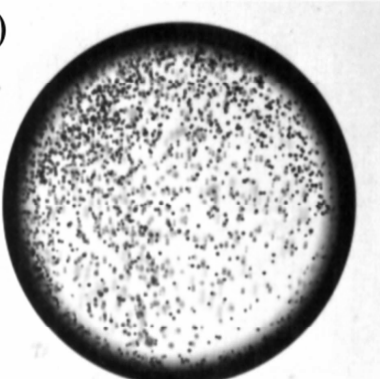

$200 \mu \mathrm{m}$

Fig. 4 Flow patterns for a droplet of DI water mixed with $0.7 \%$ w/w of $7-\mu \mathrm{m}$ fluorescent particles surrounded by mineral oil (M5904) with $1 \% \mathrm{w} / \mathrm{w}$ of Span 80 . Acoustic actuation conditions: (a) $50 \mathrm{~Hz}, \pm 85 \mathrm{~V}$ (b) $500 \mathrm{~Hz}, \pm 88 \mathrm{~V}$ (c) $1 \mathrm{kHz}, \pm 88 \mathrm{~V}$ (d) $1.5 \mathrm{kHz}, \pm 86 \mathrm{~V}$ (e) $1.6 \mathrm{kHz}$, $\pm 85 \mathrm{~V}$ (f) $2 \mathrm{kHz}, \pm 83 \mathrm{~V}$ (g) $4.5 \mathrm{kHz}, \pm 77 \mathrm{~V}$ (h) $5 \mathrm{kHz}, \pm 61 \mathrm{~V}$ (i) $6 \mathrm{kHz}, \pm 61 \mathrm{~V}$. 

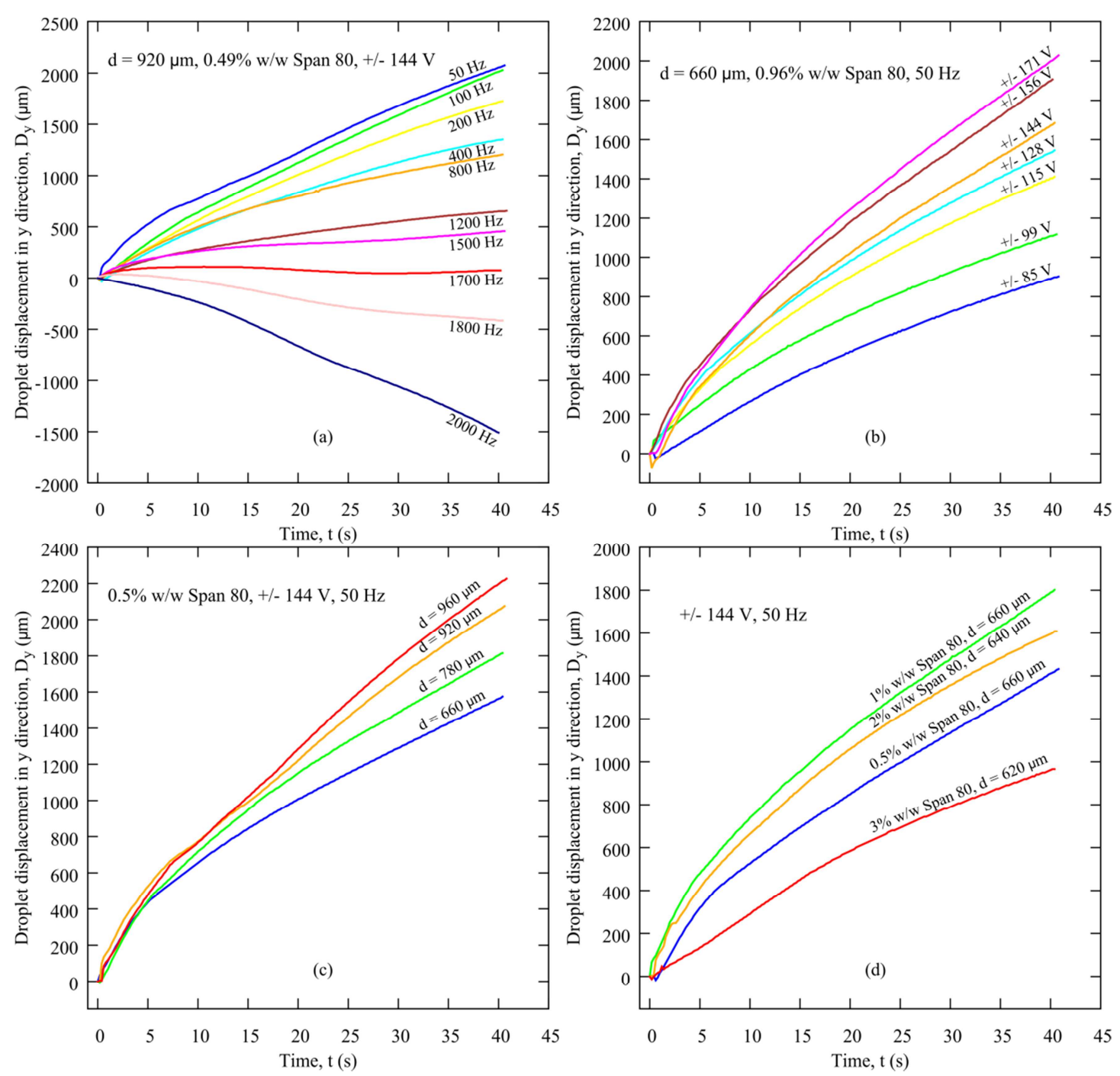

Fig. 5 Variation of droplet displacement in the $y$ direction $D_{y}$ with different experimental parameters: (a) Frequency f; (b) Voltage $V$; (c) Droplet diameter $d$; (d) Surfactant concentration. 

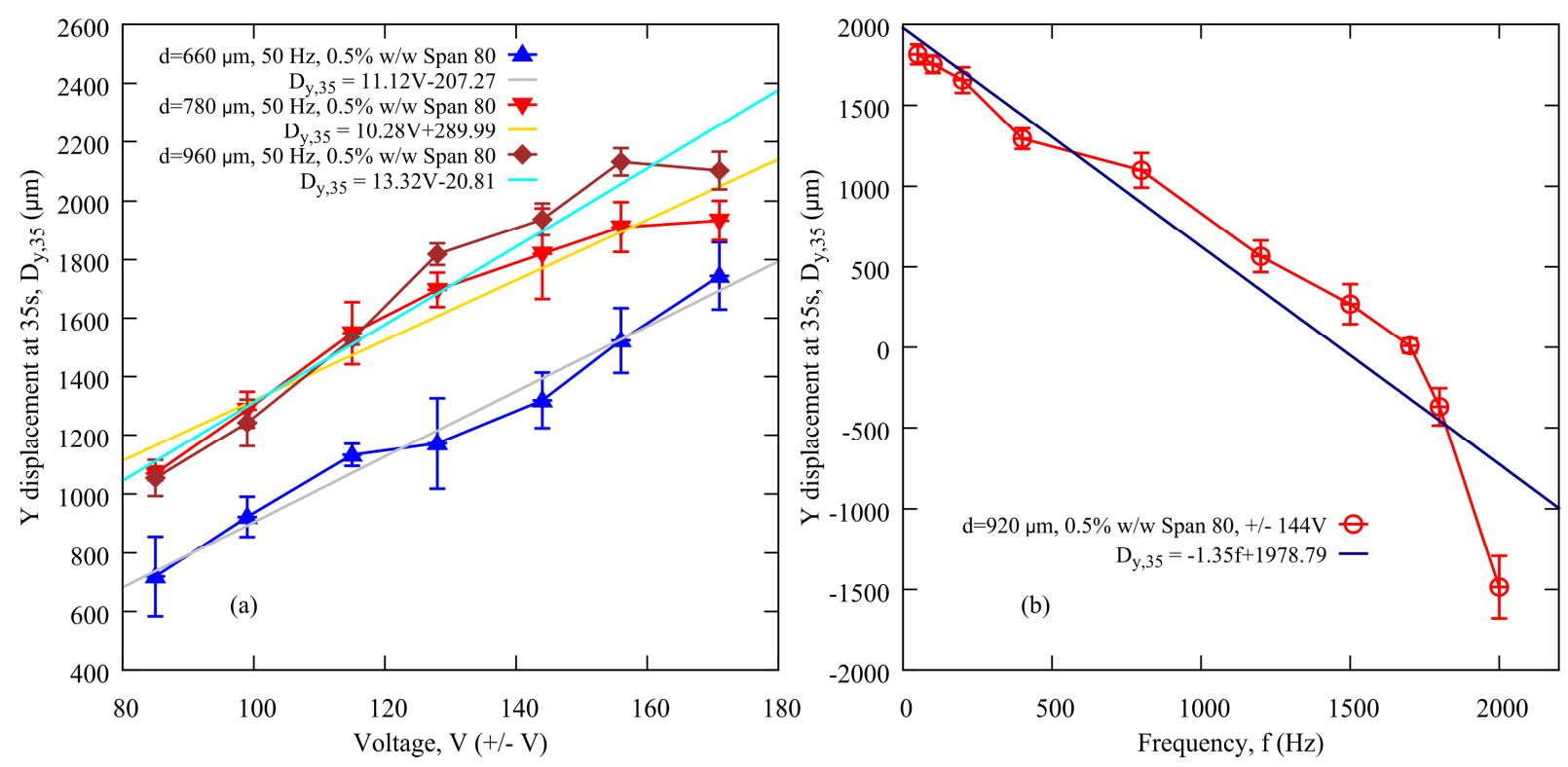

Fig. 6 Droplet displacement at $\mathrm{t}=35 \mathrm{~s}$ as a function of different parameters: (a) Voltage $V$; (b)

Frequency $f$.
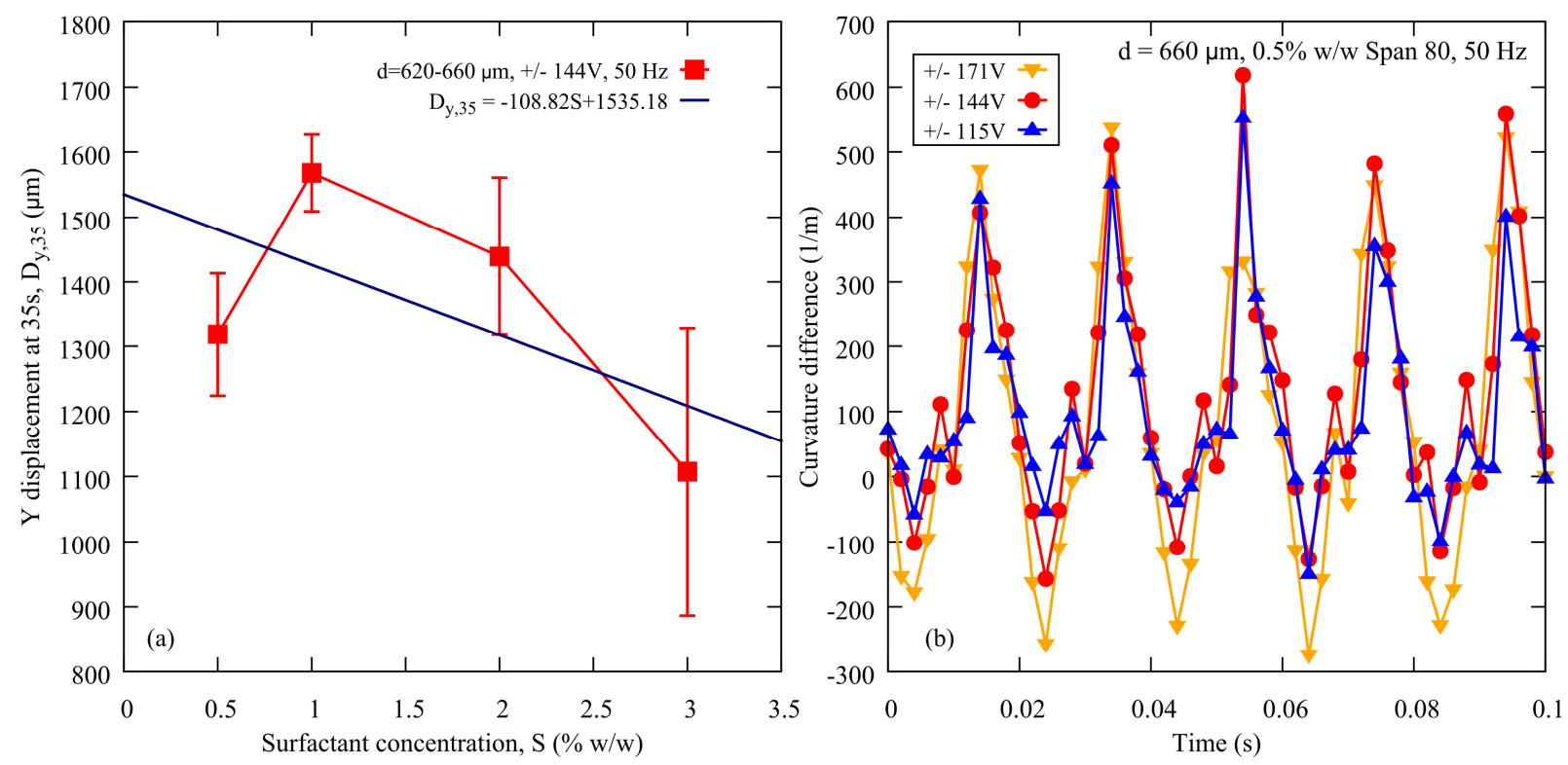

Fig. 7 (a) Droplet displacement at $t=35 \mathrm{~s}$ as a function of surfactant concentration; (b) Time variation of the curvature difference, $1 / R_{a d v}-1 / R_{r e c}$, between the front and the rear part of a droplet with diameter $d=660 \mu \mathrm{m}$ actuated at $50 \mathrm{~Hz}$ and different applied voltages. 

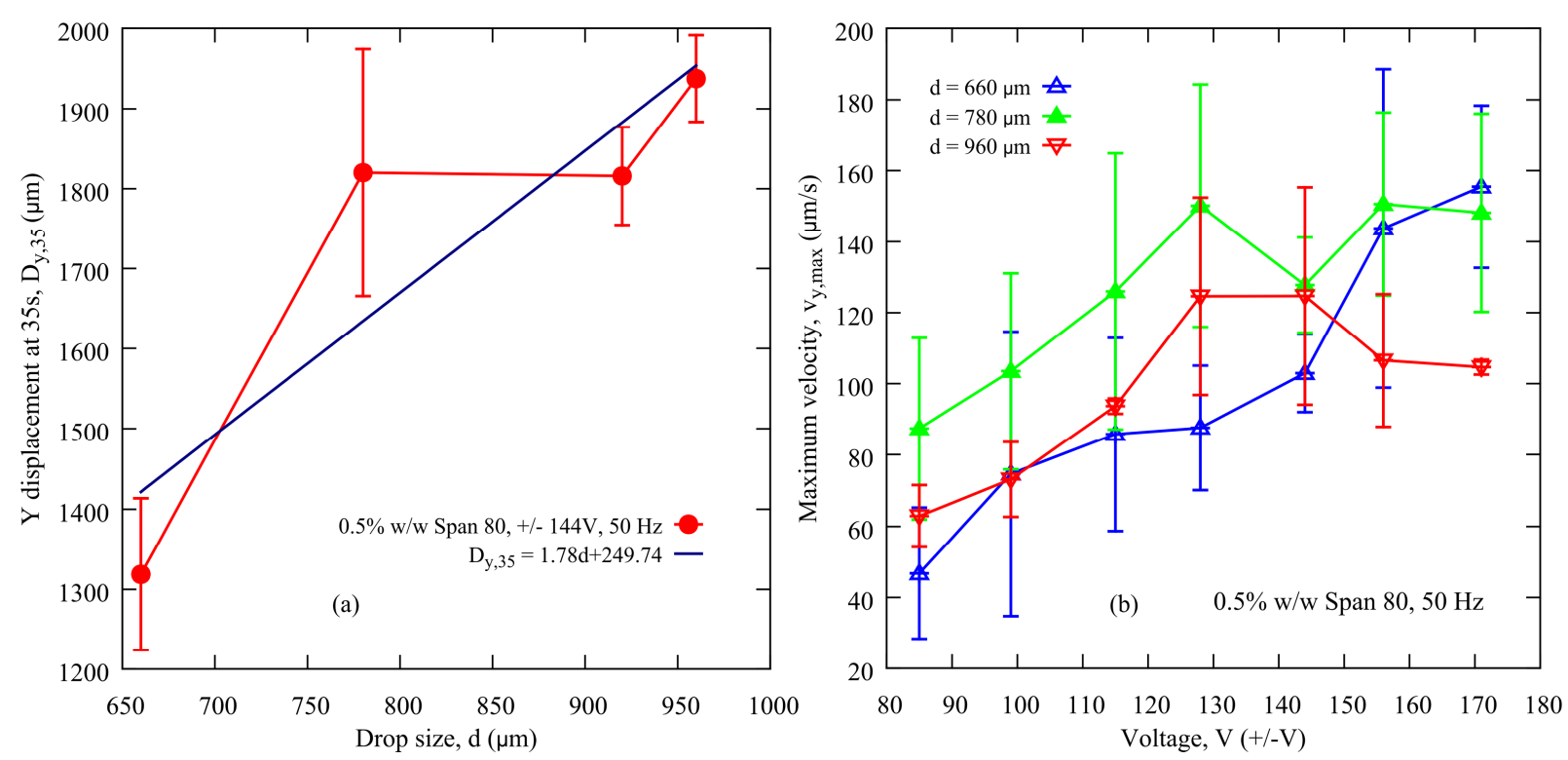

Fig. 8 (a) Droplet displacement at $t=35 \mathrm{~s}$ as a function of droplet size; (b) Maximum velocity as a function of the applied voltage $V$.

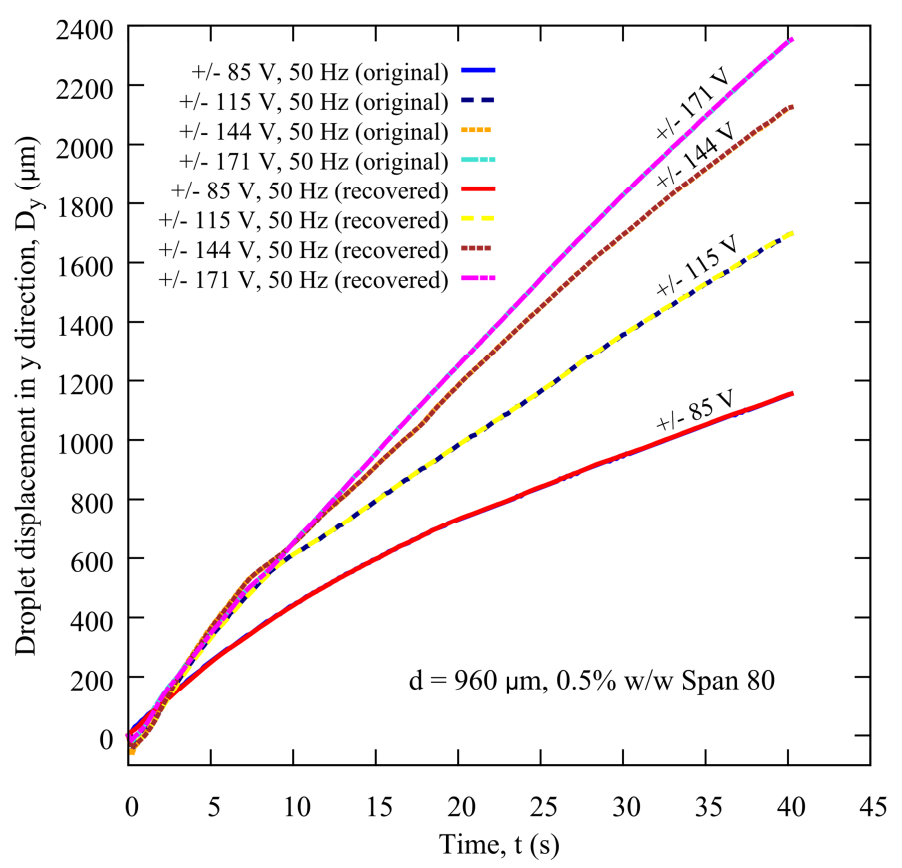

Fig. 9 The original (experimental) displacement curves and the recovered curves based on an interpolator derived from the sampling theorem for a droplet of $d=960 \mu \mathrm{m}$. 

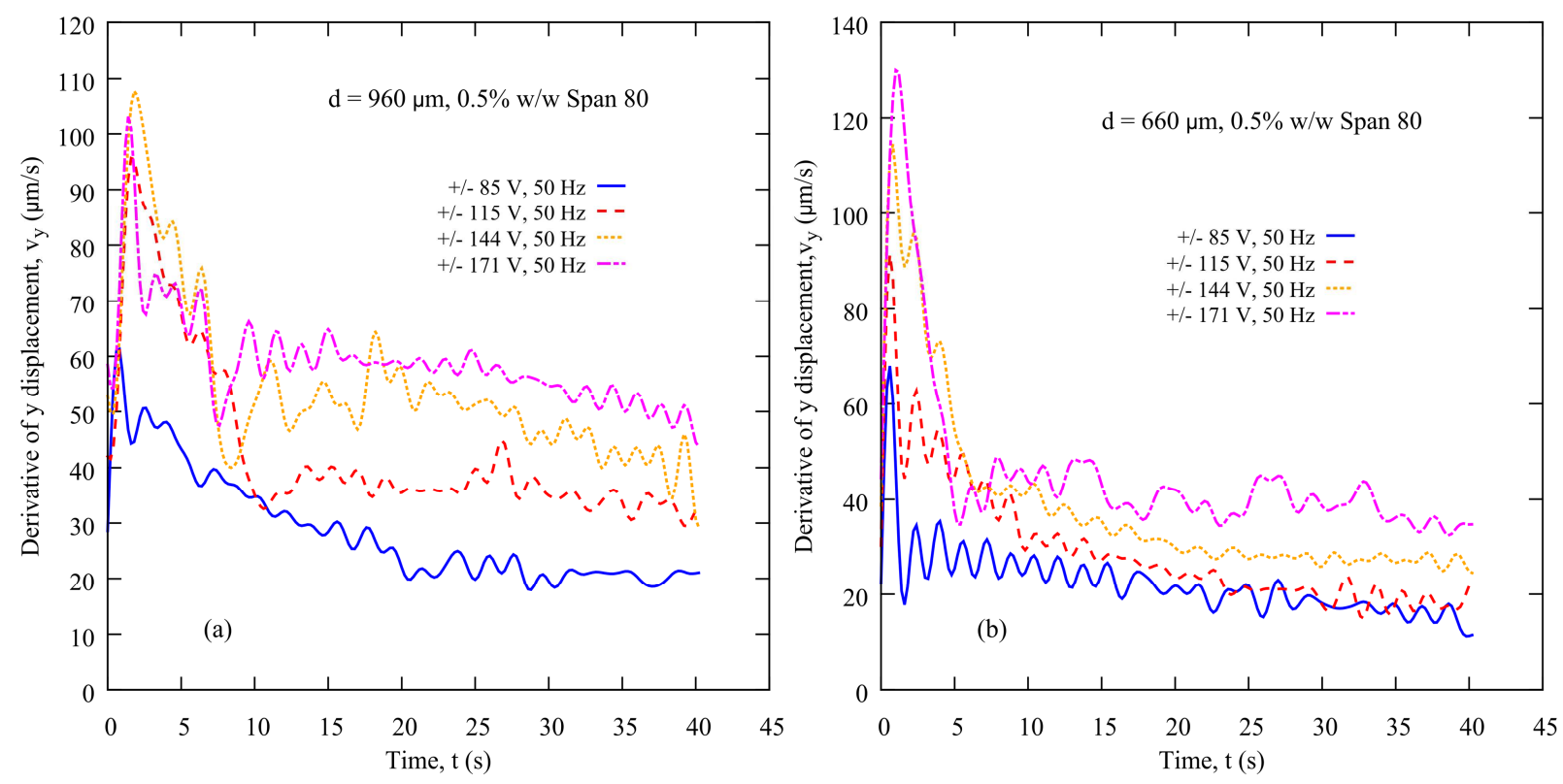

Fig. 10 Velocity $\left(v_{y}\right)$ curves of droplets with (a) $d=960 \mu \mathrm{m}$ and $(b) d=660 \mu \mathrm{m}$ under different actuation conditions.

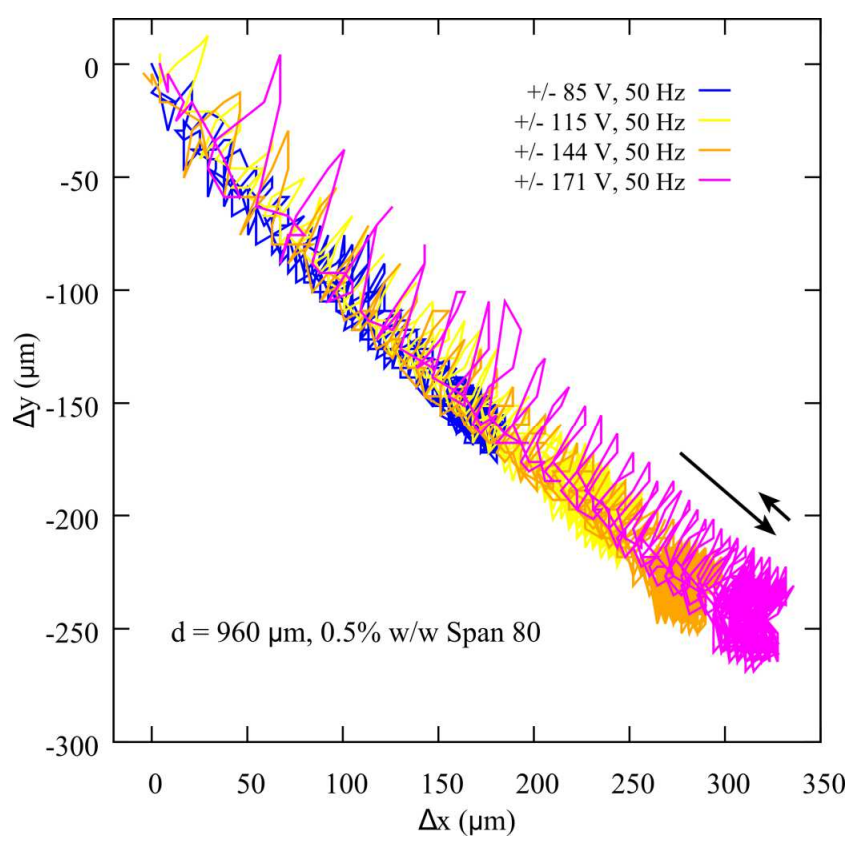

Fig. 11 Drop shape change, $\Delta x$ and $\Delta y$, with time as the running parameter for a droplet of $d=$ $960 \mu \mathrm{m}$ under different acoustic conditions. The total time duration is around $5.5 \mathrm{~s}$. 


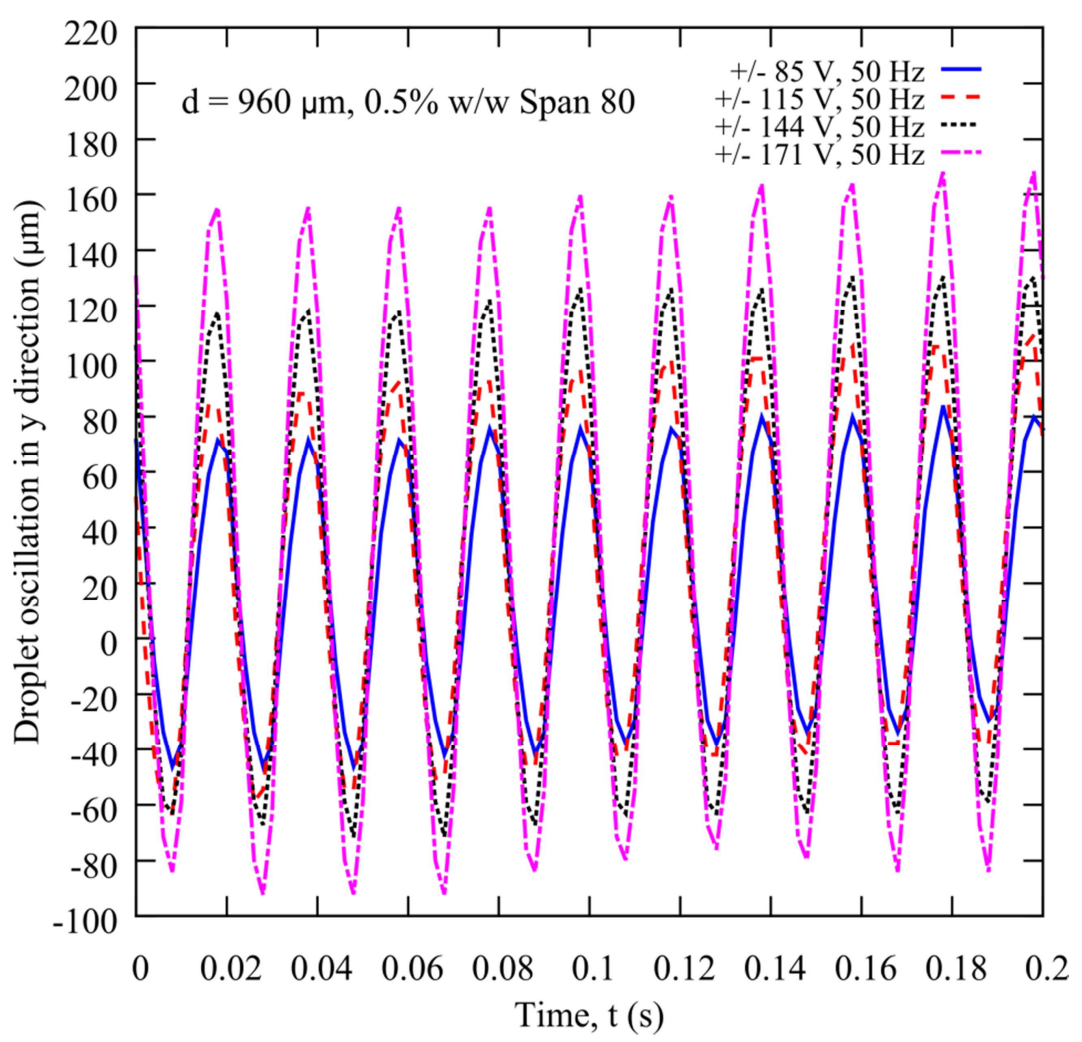

Fig. 12 Droplet oscillation in the $y$ direction, change of $y_{c}$ over time, under different acoustic actuation conditions.

Video captions:

S1 Acoustic streaming in a water droplet actuated by a voltage of $\pm 85 \mathrm{~V}$ at $50 \mathrm{~Hz}$ in mineral oil with $1 \%$ w/w Span 80.

S2 Acoustic streaming in a water droplet actuated by a voltage of $\pm 85 \mathrm{~V}$ at $1.6 \mathrm{kHz}$ in mineral oil with $1 \%$ w/w Span 80. 
S3 Acoustic streaming in a water droplet actuated by a voltage of $\pm 77 \mathrm{~V}$ at $4.5 \mathrm{kHz}$ in mineral oil with $1 \%$ w/w Span 80.

S4 Acoustic streaming in both phases actuated by a voltage of $\pm 171 \mathrm{~V}$ at $50 \mathrm{~Hz}$ in mineral oil with $1 \%$ w/w Span 80 .

S5 Acoustic streaming in both phases actuated by a voltage of $\pm 171 \mathrm{~V}$ at $2 \mathrm{kHz}$ in mineral oil with $1 \%$ w/w Span 80.

S6 Forward motion of a water droplet in mineral oil with $0.5 \%$ w/w Span 80 actuated by a voltage of $\pm 156 \mathrm{~V}$ at $50 \mathrm{~Hz}$. 
A novel droplet manipulation method using acoustic radiation pressure and acoustic streaming in a microfluidic environment. In an acoustic field, droplets deform, oscillate and move in a wide range of applied frequencies.

(a)

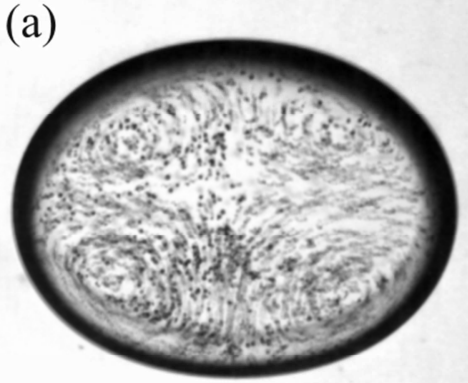

$50 \mathrm{~Hz}$

(d)

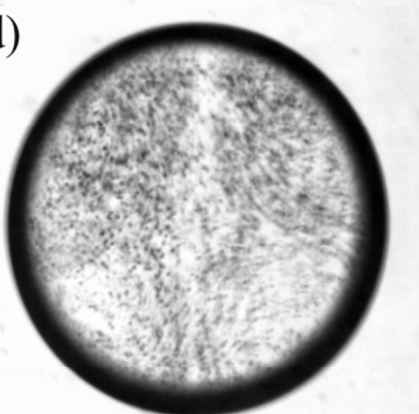

(b)

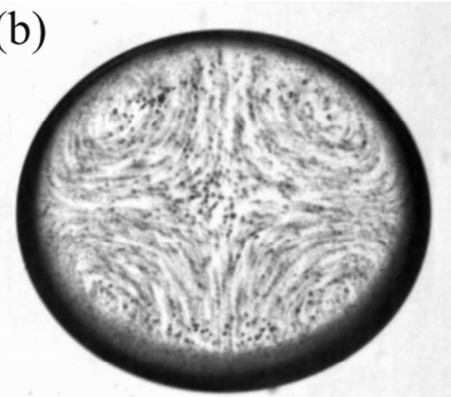

$500 \mathrm{~Hz}$

(e)

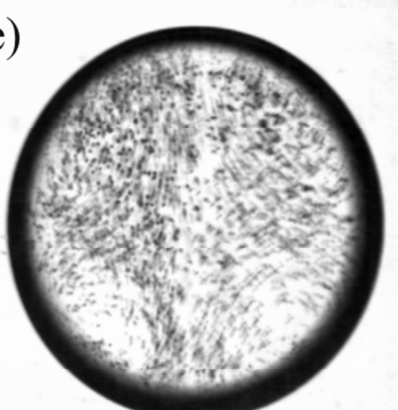

$1.6 \mathrm{kHz}$

(g)

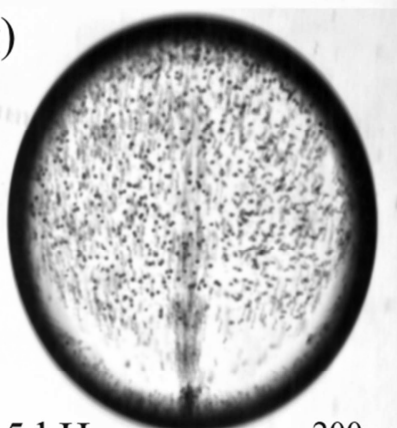

(h)

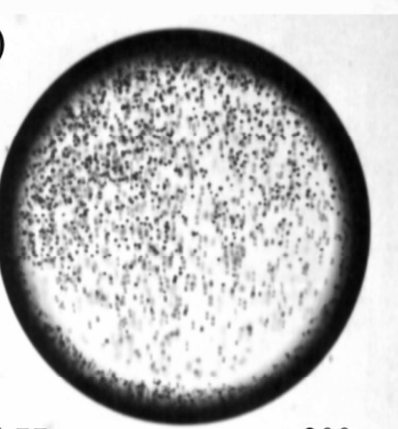

(c)

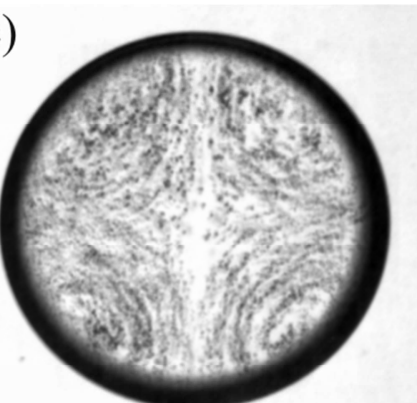

$1 \mathrm{kHz}$

$200 \mu \mathrm{m}$

(f)

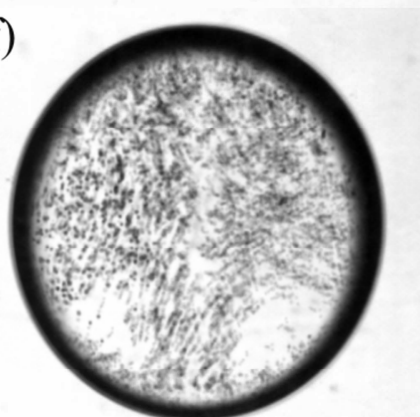

$2 \mathrm{kHz}$

$200 \mu \mathrm{m}$

(i)

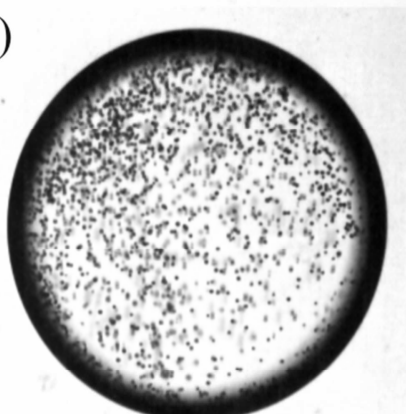

$4.5 \mathrm{kHz}$

$200 \mu \mathrm{m} \quad 5 \mathrm{kHz}$

$200 \mu \mathrm{m} \quad 6 \mathrm{kHz}$

$200 \mu \mathrm{m}$ 